Economía, Sociedad y Territorio, vol. vIII, núm. 27, 2008, 809-816

\title{
El espacio infinito recuperado
}

\section{Recovered infinite space}

\author{
Cisneros Sosa, Armando (2006), El sentido del espacio, Porrúa, \\ MÉXICO, 131 PP., ISBN: 970-701-829-1.
}

En los inicios del siglo xxI existen temas y problemas que no pierden su importancia y que con el avance de la ciencia y las nuevas condiciones sociales de la posmodernidad readquieren actualidad y conllevan polémica y debate. Tal es el caso de la categoría espacio -primordial tanto para las ciencias fisicomatemáticas como para las ciencias sociales-, cuyo significado y utilización ameritan reflexión y revisión, sobre todo de carácter epistemológico, más ahora que la tendencia en el desarrollo científico es a la integración e hibridación de los conocimientos en aras de acceder a la complejidad de los procesos y avanzar así en su explicación y comprensión.

¿Qué es el espacio?, se pregunta el autor del texto que nos ocupa, como una interrogante que lo lleva a un recorrido filosófico desde Platón hasta Merleau-Ponty (1961), inquiriendo en todo momento acerca de los fundamentos científicos que soportan las diferentes ideas y explicaciones elaboradas en torno al espacio y su entendimiento. $Y$ ese es precisamente uno de los atractivos de la lectura en cuestión, que nos recuerda la importancia de acudir a los autores originales y clásicos cuando indagamos sobre el sentido de algún objeto de estudio, y más aún si éste no tiene una sola significación ni su ámbito se restringe a un campo de conocimiento, sino que se trata de una categoría general que presenta varias dimensiones y diferentes disciplinas la asumen como básica dentro de sus teorías y supuestos epistemológicos.

Así que iniciando con el gran Platón, y enmarcado en la soberbia cultura griega donde se iniciara en buena medida la ciencia occidental, encontramos la idea del espacio como el lugar natural del mundo, concebido como espacio-contenido y a la vez continente de los objetos de la realidad; relación entre lo sensible y lo inteligible, entre lo visible y lo invisible. Se trataba de una complejidad que por lo mismo se remitía a una incógnita y una noción derivada de la percepción sensible a partir de las ideas.

En cambio Aristóteles, discípulo de aquél y fundador de varias disciplinas, desde su visión de científico empírico e innova- 
dor afirmó que el espacio era principalmente un medio, el principal ciertamente que daba cuenta de la proporción de los movimientos y la fuerza de los cuerpos, es decir, el espacio lugar que sin embargo no coincide con la materia -como había creído Platón- ni con la forma de los cuerpos particulares y tampoco con la distancia entre ellos. Es más bien el límite del cuerpo envolvente respecto del cuerpo envuelto. En efecto, Aristóteles representa a todos los cuerpos como envueltos por otros cuerpos y así surge el lugar (lugar individual). También el mundo en su conjunto está rodeado y ceñido por un límite, el primer cielo, y así surge el espacio general. De ahí que no existe el espacio vacío, por ello se concibe de forma enteramente realista: todo está lleno de cuerpos, no existen espacios intermedios vacíos. Además, el espacio es algo estable, por ello es que puede haber un arriba y un abajo y así también puede darse un movimiento, toda vez que únicamente así se puede lograr un contacto.

Siguiendo la historia del pensamiento filosófico-científico, cruzamos ahora por el Renacimiento y los nuevos caminos de la ciencia, para detenernos en la trascendente figura de Galileo, quien desarrolla la idea del espacio mecánico, útil, observable con toda precisión, incluso matematizable, aportando axiomas demostrables por medio del experimento y, sobre todo, a través de la lógica y la geometría. El punto fuerte radica en la afirmación de que el espacio se puede representar, calcular y manejar científicamente - a partir del estudio de sus tres dimensiones- y de esta manera entender y medir con exactitud el movimiento uniforme, la velocidad, la distancia y los tiempos. Ahí quedaba fijada una de las concepciones medulares del espacio que sentaban las bases para todo el desarrollo posterior de las disciplinas científicas sustentadas en la geometría y el cálculo matemático del espacio y sus complejidades, así como los fundamentos de la física moderna.

Avanzando por los caminos del racionalismo, y reconociendo la importancia de Descartes como padre de la filosofía moderna, se recupera la idea del espacio lugar, pero asimismo de la res extensa como objetos susceptibles de la mathesis, encontrando que en la naturaleza y sus movimientos en el espacio dominaba una mecánica rigurosa y precisa, con la función de continuar en todo momento en línea recta y sin contratiempos. Entonces, en el mundo de los cuerpos, es decir, de todo cuanto puede ser limitado por alguna figura, circunscrito en un lugar y que llena un espacio excluyente de otro cuerpo -que se puede sentir y mover de varias maneras menos por sí mismo-, se impone la matematización y la 
concepción del cuerpo como espacio lleno, donde esa sustancia amplia se extiende en longitud, anchura y profundidad.

De ahí le siguió Newton en ese camino, reafirmando la tesis del espacio como premisa y objeto de la razón moderna, surgido de la evidencia del razonamiento lógico, de una intelección superior a las evidencias empíricas y a la imaginación. Estamos ante el predominio de una razón sustentada en las matemáticas basadas en un método riguroso y exacto de observación de las evidencias, que procede a dividir los problemas en partes y los contabiliza, concibiendo a éste como el único método seguro que podría explicar todas las cosas del Universo, incluyendo las pasiones del alma, que a partir de ese momento formarán parte del mundo material y, por lo mismo, se les considera plenamente orgánicas.

En seguida Armando Cisneros hace una parada a la vez obligada y crucial para su análisis, ya que aborda las profundas reflexiones de Immanuel Kant -eminente representante de la Ilustración alemana y del propio idealismo alemán- para quien se trataba, ante todo, del espacio de la razón pura, fiel instrumento de conocimiento; si bien ligado estrechamente a la sensibilidad, más que todo lo concebía como instrumento epistemológico. No obstante, sin desconocer los avances de la física de su tiempo, también lo encontraba móvil y relativo, espacio que mueven otros espacios, por lo tanto espacios-lugares, demostrables empíricamente. Asimismo, espacio infinito, puro, a priori y necesario. Sin duda la referencia kantiana resulta fundamental no sólo por la reivindicación del espacio (físico) lugar, sino, y aún más, por la amplitud de sentido que representó la idea del espacio como categoría a priori del entendimiento, de la mano de la categoría de tiempo, de la cual definitivamente no podría aislarse, como por la enorme vía de interpretación que significaba el espacio pensado, no únicamente representado, sino con una existencia real en el pensamiento, además de las dos dimensiones trascendentales que concibió: espacio infinito, puro; y espacio necesario, condición de la existencia de los objetos en el mundo. Puede entonces hablarse del espacio pensante, donde la razón pura trasciende los meros sentidos.

De ahí un gran salto hasta la fenomenología, desde luego en la persona y obra de Edmund Husserl, en quien la concepción del espacio habría de cambiar radicalmente a partir de un cuestionamiento de fondo a la validez absoluta de las representaciones del espacio moderno. Es así que desde las entrañas del razonamien- 
to matemático, este gran pensador y filósofo orienta su reflexión hacia la idea de la espacialidad, misma que trasciende los conocimientos de las ciencias -tanto de la física como de la geometríapara asentarse plenamente en la naturaleza pensante; si bien a la vez sobre la base del mundo concreto. La dimensión eminentemente humana de la espacialidad haría que Husserl buscara abarcar la conciencia de lo vivido, que lo llevaría a una filosofía de las ciencias humanas, deslindando asimismo la perspectiva psicológica, a fin de mantener la evidencia de la materia pero sin desconocer el mundo subjetivo. Ese mundo de la vida que gira en torno a la espacialidad, la de los cuerpos desde donde percibimos y nos ubicamos en el mundo, con su temporalidad, el lenguaje y las cosas cotidianas. Entonces quedaban relativizadas la ciencia y la modernidad, acotadas a su época y sujetas a la crítica que se orientaba a la denuncia de tendencias positivistas mecanicistas y por lo mismo reduccionistas, que no dejaban de pretender imponer la concepción del espacio físico como la única y verdaderamente válida. En contraposición a esta visión limitada, la fenomenología estableció la prioridad del espacio del mundo vital, donde lo más importante es percibir la esencia de la conciencia, en todas y cada una de sus manifestaciones. Entonces, este espacio, el del mundo de vida representado con cuerpos en reposo y movimiento no es, a pesar de su apariencia, el mismo que el espacio de la física, sino el de la experiencia cotidiana, donde las experiencias interconectadas se traducen en representaciones y conforman ideas vigentes sobre el espacio.

Seguramente había nacido la posmodernidad, de ahí que el concepto de espacio aparece con un tono revolucionario. No es ya el espacio objetivista de la física ni de todas las otras ciencias surgidas en el siglo XIX -considerando a las humanas- y menos aún el espacio de la razón pura, o un espacio relativista empiriocriticista. No, ahora el espacio es completamente humano, un espacio mucho más vasto, asentado en la subjetividad, específicamente en su parte no intelectual y ligado al mundo vivido del sujeto. Se trata de un espacio relativo, según cada sujeto, plenamente representado: un espacio mundano, precientífico que no necesita considerarse como objeto físico observable y medible.

Es entonces cuando Cisneros enlaza a Husserl con la filosofía de la existencia de Heidegger, que en su visión ontológica de la existencia postula la tesis del ser como Ser-en-el-mundo, equivalente a, estar en compañía de, anticiparse a sí mismo. Es su perspectiva del espacio-tiempo, teniendo como referencia la revolu- 
ción de Einstein en la física, la filosofía y en general la cultura y concepción de la vida y de los hombres, pero especialmente la tesis del binomio espacio-tiempo, antes vistos como separados, quedaba en claro que ahora la percepción del espacio dependía del observador, no podía ser otra sino la de una espacialidad, ante todo, plenamente humana y por lo mismo útil para el ser, que resultaba objetiva y subjetiva al mismo tiempo. También ligada estrechamente a la corporeidad, a las cosas, el orden y culturalmente determinada donde, por lo mismo, las distancias y el tiempo son relativos. Llegamos así a la reflexión ontológica de un espacio del ser, en que el espacio era tanto el soporte de las representaciones como de las cosas concretas, el detentador de las cosas del exterior. Así, esa espacialidad, diferente del espacio físico, es la del ser, por lo que el espacio no será meramente intelectivo (Kant) sino acorde con Husserl, físico y subjetivo, de representaciones y cosas representadas. Es el espacio de la existencia, profundamente fenomenológico, es decir, resultado de la conciencia de las cosas a partir de la intencionalidad que la orienta. Sin embargo, en la visión heideggeriana del espacio, las cosas ocupan un lugar central, ya que no puede hablarse del espacio vacío, sino de un espacio como mundo circundante, lleno de cosas; pero sobre todo, de cosas significativas para el sujeto, que ocupan un lugar no físico sino en las preferencias del mismo. Vemos así la idea de una espacialidad relativa y eminentemente subjetiva, surgida esta concepción más como una crítica de las visiones cientificistas y realistas, donde lo más importante, incluso que la ciencia, es tener al tiempo como crucial para el hombre y al espacio como un determinante primordial de la vida social.

A partir de esa consideración, la interpretación se dirige naturalmente a la postura y trabajos de Maurice Merleau-Ponty, quien asumió en forma radical una crítica de la modernidad de orientación fenomenológica. Este autor francés desarrolla una concepción del mundo vital extenso, concreta y abstracta a la vez, parte de un mundo que es representante y representado, significante y significado, dualidad movida por la intencionalidad del sujeto. Se trata de captar lo concreto y lo que se piensa de él, entonces un espacio representado, ya sea como reflejo de lo objetivo o virtual, sobre el sujeto y su mirada. Es un espacio no sólo humano sino profundamente corporal, cinestésico, sujeto a las coordenadas de los órganos, nunca homogéneo y ligado directamente a la geometría existencial. En sí mismo espacio-ser 
vinculado a la concepción del mundo vital-complejo. También en Merleau-Ponty, y tal vez con mayor insistencia, se da una relativización del saber científico derivada de una concepción posmoderna del mundo, del hombre y de su espacio que es, plenamente, espacio vital. Se trata del espacio como el gran lugar del universo posmatematizable, sitio de todas las cosas, por lo tanto nunca vacío sino lleno de significaciones, de cosas con edad y dimensión, incluso con una historia. El espacio existencial, por tanto, no puede ser meramente una especie de éter en el que todo aparece ni una serie de disposiciones lógicas, incluso axiomáticas, como materia de la geometría. El espacio tiene que ser vivo, tanto concreto y conceptual como experimentado. Finalmente, el espacio es tal cual aparece, incierto pero vigente en la piel, claro a la mirada y al tacto.

Pretendiendo una mínima ponderación sobre la obra de Armando Cisneros, apuntaré las siguientes ideas acerca de su pertinencia e importancia:

1) El texto resignifica una categoría central como es la de espacio y la aborda desde una mirada posmoderna, en la cual integra en un mismo esquema analítico y de interpretación las distintas visiones generadas por otras tantas disciplinas a través de la historia del pensamiento moderno y su crítica.

2) Invita a reflexionar, sentir y vivir el espacio, a la vez que refrenda su materialidad y subjetividad en una hibridación que puede captarse cuando se trascienden los límites de la ciencia positivista y se alcanza la mirada sin límites de las múltiples dimensiones de lo experimentado, lo necesario y lo relativo.

3) Esta lectura expresa y marca una inquietud por ir más allá de las verdades disciplinarias, lineales y en apariencia plenamente legitimadas, donde lo más importante es buscar con rigor y apertura a la vez la diversidad y la diferencia como vías de reflexión y encuentro con lo que no se deja sujetar en esquemas rígidos y unívocos, porque en su esencia está el no ser del todo abarcables, comprensibles ni definidos, y en ello va su trascendencia.

4) Expresa y motiva una revaloración de la filosofía, así como un abordaje interdisciplinario que permita enfocar la interpretación a partir de una operación de abstracción, indispensable para poder pensar una categoría de tal grado de generalidad y complejidad. 
5) Asimismo, el trabajo resume, esclarece y da perspectivas de estudio y líneas de análisis que, desde la fenomenología, podrían aportar cauce a nuevas indagaciones, caminos seguramente iniciados; pero que sin fin muestran nuevas caras y en su devenir asomarán otras miradas.

6) Para las ciencias sociales, una reflexión así representa un aire fresco, un pretexto para volver a pensar sobre el espacio, en realidad los espacios, sobre todo el social, que se advierte como previo y determinante de la ocupación de los lugares físicos; aunque sin dejar de verlo como historia personal y subjetiva, en los mundos de vida, espacio social que da sentido y en el que la vida discurre y se reinventa.

7) $\mathrm{Al}$ atardecer de la lectura, uno no puede dejar de pensar y sentir que, efectivamente, el espacio está ahí; pero igualmente es infinito; que lo requerimos como algo dado y que sin embargo lo construimos con nuestra propia vida que no deja de perseguirlo por todas partes como a su misma alma que es errante y evasiva, al tiempo que se anida en lo más íntimo de las vivencias, objetos y caminos que sin cesar inventamos para seguir por siempre entre palabras y sentidos sin reposo.

José María Aranda Sánchez Universidad Autónoma del Estado de México Correo-e: aranda@uaemex.mx, chemaaranda@gmail.com

Recibido: 3 de julio de 2007. Aceptado: 6 de julio de 2007.

José María Aranda Sánchez. Es licenciado en psicología por la Universidad Nacional Autónoma de México (UNAM); maestro en sociología por la Universidad Autónoma del Estado de México (UAEM) y doctor en urbanismo por la UNAM. Actualmente es profesor e investigador de tiempo completo del Centro de Investigación en Ciencias Sociales y Humanidades (CicsyH) de la UAEM. Sus líneas de investigación son: movimientos sociales en México y organizaciones de la sociedad civil. Es líder del cuerpo académico Investigación en ciencias sociales y humanidades del cicsyH; integrante del comité editorial de la revista Contribuciones desde Coatepec y miembro del Sistema Nacional de Investigadores (SNI) nivel I. Entre sus publicaciones recientes se 
encuentran: "Redes sociales y reflexividad: su importancia en la construcción de la sociedad civil en México", Ciencia Ergo Sum, 14(2), UAEM, México, pp. 141-150 (2007); "Perspectiva de género para el análisis de la participación femenina en organizaciones ambientalistas: el caso de la organización de mujeres ecologistas de la sierra de Petatlán, Gro.”, Territorios, 16-17, enero-julio, 2007, publicación realizada conjuntamente por la Asociación Colombiana de Investigadores Urbanos Regionales (ACIUR), Centro de Estudios Políticos e Internacionales de las Facultades de Ciencia Política, Gobierno y Relaciones Internacionales de la Universidad de Rosario, Bogotá, Colombia, Editorial Universidad del Rosario Colombia, pp. 107-125. 